\title{
Theory and simulation of gelation, arrest and yielding in attracting colloids
}

\author{
M E Cates ${ }^{1}$, M Fuchs ${ }^{2}$, K Kroy ${ }^{3}$, W C K Poon ${ }^{1}$ and A M Puertas ${ }^{4}$ \\ ${ }^{1}$ School of Physics, JCMB Kings Buildings, The University of Edinburgh, Mayfield Road, \\ Edinburgh EH9 3JZ, UK \\ ${ }^{2}$ Fachbereich Physik, Universitaet Konstanz, D-78457 Konstanz, Germany \\ ${ }^{3}$ Hahn-Meitner Institut, Glienicker Straße 100, 14109 Berlin, Germany \\ ${ }^{4}$ Group of Complex Fluid Physics, Department of Applied Physics, University of Almeira, \\ 04120 Almeira, Spain
}

\begin{abstract}
We present some recent theory and simulation results addressing the phenomena of colloidal gelation at both high and low volume fractions, in the presence of short-range attractive interactions. We discuss the ability of mode-coupling theory and its adaptations to address situations with strong heterogeneity in density and/or dynamics. We include a discussion of the effect of attractions on the shear-thinning and yield behaviour under flow.
\end{abstract}

\section{Introduction}

Recent studies of mode-coupling theory (MCT) have predicted various kinetic arrest scenarios for colloids with short-range attractions [1-3]. The behaviour at the arrest transition has been analysed in considerable detail as a function of the volume fraction $\phi$ of the colloids, their attraction range $\delta$ (in units of the particle radius $a$ ) and the attraction $\varepsilon$ (in units of $\left.k_{\mathrm{B}} T\right)[4,5]$. This approach, which builds on a successful theory of the glass transition for hard spheres, suggests that the underlying 'ideal glass transition', found within MCT, could provide a universal mechanism for homogeneous gelation in which the arrested state is viewed as an attraction-driven glass $[6,7]$.

Here we do not attempt to mediate between those who support and those who oppose the basic philosophy of MCT (see [8,9] for discussions). Instead, in sections $2-4$, we outline three areas of recent work that examine the ability of MCT to deal with heterogeneous systems. In section 2 we consider attractive colloids at low density, and consider kinetic arrest by routes that combine aspects of irreversible aggregation, gelation, and phase separation. The phenomenology that we describe takes MCT as a reliable theory of arrest at high densities only, and attempts to extend the same picture to the much lower colloid volume fractions at which gelation actually occurs in systems with strong short-range attractions. The resulting gels are very heterogeneous and our approach is to preserve what we can of the MCT theory while allowing for heterogeneity in density at scales either shorter or longer than those at which

Konstanzer Online-Publikations-System (KOPS)

URL: http://www.ub.uni-konstanz.de/kops/volltexte/2007/3794/

URN: http://nbn-resolving.de/urn:nbn:de:bsz:352-opus-37949 
we apply the MCT. In the first case (shorter-scale heterogeneity) one has a picture involving the MCT-like arrest of clusters; in the second (longer-scale) one has a phase-separation driven morphology in which one phase arrests into a dense gel or attractive glass.

In section 3 we turn to gelation at higher particle densities, where unmodified MCT can reasonably hope to succeed, at least in the absence of phase separation. Here we carefully analyse simulation data for attractive colloids, suppressing phase separation by including a weak, long-range repulsion. We find that MCT gives an excellent account of averaged dynamical quantities but that this disguises an underlying dynamics which is much richer, and which in fact shows many hallmarks of dynamical heterogeneity (DH): populations of fast- and slow-moving particles coexist. This heterogeneity appears to be closely related to static heterogeneity of density which is present for attractive colloids even at quite high volume fractions. This form of DH may thus be different from that found in hard spheres [10] where the density fluctuations are smaller. The new type of DH may be enhanced by the long-range repulsion we have added, but even if that turns out to be true, the results are of strong interest. They show firstly that when experimental data agree with MCT this does not exclude the possibility of DH, likewise that observation of DH does not exclude the possibility that MCT remains predictive for averaged quantities such as the dynamic structure factor on the fluid side of the transition.

In section 4 we describe some related recent work on colloids under shear, and give a preliminary account of calculations that address the effect of short-range attractions on the rheological behaviour. This offers nontrivial predictions, in particular for the variation of yield stress and shear thinning behaviour with the range of attraction $\delta$. In section 5 we offer some concluding remarks.

\section{Gelation at low density}

\subsection{MCT, phase separation, and aggregation}

For attractive colloids, MCT appears to be very useful at predicting averaged dynamical quantities like the dynamic structure factor $S(q, t)$, so long as the volume fraction $\phi$ of colloid is high enough $[7,11,12]$. In the following we refer to the resulting spatially homogenous gel as type I. In practice, for moderately short relative range of attraction $\delta \gtrsim 10^{-2}$ a metastable gas-liquid phase separation [13] interferes with this kinetic arrest scenario. On quenching to create a gel, phase separation is likely to intervene for all colloid volume fractions below the intersection of the binodal with the MCT arrest line.

The 'hidden' binodal, when present, dominates over the much slower crystallization route to the thermodynamic ground state, so that it is liquid-gas separation not crystallization that can interfere more strongly with glass formation. Moreover, due to the kinetic (as opposed to thermodynamic) mechanism underlying the arrest transition, this potentially complex interplay sensitively depends on the quench rate relative to the typical timescale for phase separation. To a first approximation, the latter is given by the Smoluchowski time, which is the timescale for doublets to form by colloidal collisions. Depending on whether the quench is fast or slow with respect to this natural timescale, we expect transient gels to develop along one of two different routes, in the following referred to as type II and type III, respectively. Type-II gels are homogeneous on short scales but strongly heterogeneous at the mesoscale: they result when the characteristic coarsening textures produced by phase separation get 'frozen in' during the coarsening process, as a result of an MCT-like arrest of one of the two phases [14]. Type-III gels are, in contrast, assemblies of long-lived nonequilibrium structures locally resembling those obtained from irreversible cluster aggregation [15] and thus heterogeneous also on short scales. 


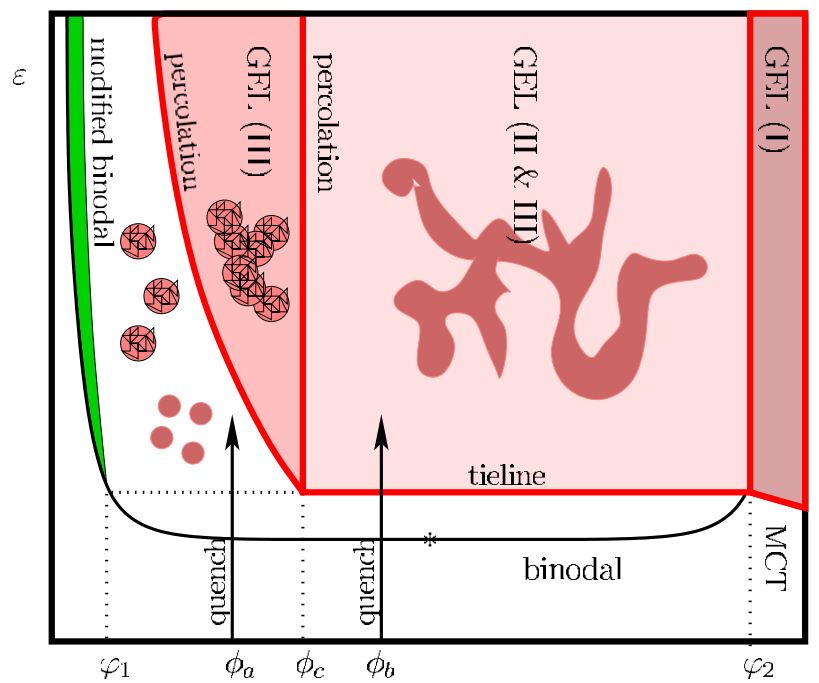

Figure 1. Schematic $(\phi-\varepsilon)$ cut through the phase diagram of adhesive hard spheres. The interaction of gas-liquid phase separation with MCT kinetic arrest gives rise to a variety of metastable gel phases. At high colloid volume fractions $\phi$, where MCT can be applied directly, homogeneous gels form (region I). At lower $\phi$ phase separation may create macroscopically heterogeneous gels (region II) and (homogeneous) gel beads if the quench is slow, while more tenuous gels (III) and (ramified) clusters consisting of nonequilibrium particle aggregates result from rapid quenches.

(This figure is in colour only in the electronic version)

To distinguish from fully irreversible aggregates, in which the bonds formed are permanent, the type III process is sometimes called 'weak gelation'.

During the process leading to type-II gels, the system remains in (or sufficiently close to) local equilibrium, so that gelation by this route represents a relatively straightforward combination of phase separation and MCT. However, additional concepts are needed for the type-III scenario. Some of us have recently proposed a schematic description (called cluster-MCT or CMCT) [16] for weak gelation in such suspensions, in terms of an effective theory for a fluid of 'renormalized particles'. These represent coarse-grained clusters and their interactions are analysed, in an MCT-like fashion, to predict the onset of global kinetic arrest. The underlying view of gelation as a double ergodicity breaking (on the monomer scale and on the cluster scale) seems to be supported by recent numerical simulations [17].

\subsection{Schematic nonequilibrium phase diagram}

A schematic phase diagram of the different nonequilibrium behaviours predicted for hardsphere colloids with short-range attractions is provided in figure 1.

Slow quench scenario. We concentrate first on this conceptually simple case, which results in type-II gels. Consider a slow quench along the quench path indicated by the arrow at volume fraction $\phi_{a}$. Upon crossing the (metastable) liquid-gas binodal, the fluid starts to decompose into a minority phase consisting of colloidal 'liquid' domains (rich in particles), and a majority 'gas' phase consisting of domains where colloids are scarce. These domains undergo slow coarsening, while their compositions continue to evolve along the binodal in response to the ongoing quench. During this process, the two phases both remain in local 
coexistence on the binodal and move apart until the compositions $\varphi_{1}$ and $\varphi_{2}$ are reached. Note that, although the quench is slow, we assume that macroscopic phase separation is even slower, so that the morphology is one of mesoscopic domains. Once the colloid volume fraction within the minority phase reaches $\varphi_{2}$ (see figure 1), which is where the binodal cuts the MCT line, the denser domains undergo kinetic arrest: the characteristic timescales for further structural evolution suddenly increase dramatically. From the lever rule, the volume fraction of space occupied by the resulting amorphous solid phase is $\Phi(\phi)=\left(\phi-\varphi_{1}\right) /\left(\varphi_{2}-\varphi_{1}\right)$.

Depending on the mesoscale domain structure that has accompanied the preceding phase separation, this volume fraction $\Phi$ may be more or less than the value $\Phi_{c}$ required for the minority phase to percolate. Only if it does percolate do we have a macroscopic solid or gel: note that gelation, in the sense of a finite elastic modulus, requires percolation of an arrested phase, or equivalently arrest of a percolated one. In particular, existence in the system of a percolating network of bonds, each of which is transient, is not sufficient to create a modulus.

In terms of the colloid volume fraction $\phi$, we obtain a percolation threshold $\phi_{c}$ defined by $\Phi\left(\phi_{c}\right)=\Phi_{c}$. For a volume fraction $\phi_{b}>\phi_{c}$ as depicted in the figure, a connected gel, of finite modulus, is the predicted result of a quench. By varying the colloid volume fraction and the quench kinetics, different gel morphologies should be realizable in a relatively well controlled manner. Indeed, corresponding recipes are routinely applied in the industrial processing of colloidal and polymeric gels for optimizing rheological properties (see e.g. $[18,19])$. Upon decreasing $\phi$, the gel gets more tenuous and eventually, for $\phi<\phi_{c}$ (e.g., $\phi=\phi_{a}$ in figure 1), this disintegrates into a fluid of slightly sticky gel beads. (At still lower densities, there is a slight shift in the binodal curve because the coexisting dense phase cannot be denser than $\phi_{2}$.)

For moderately deep quenches, the mutual attraction between the colloids, though strong enough to solidify dense colloidal drops (forming the aforementioned beads), is insufficient to permanently bind beads that come into contact. This is because the beads are now made of colloidal glass, and cannot adapt their shapes to allow coalescence on the Smoluchowski timescale, even though this would reduce their surface energy significantly. Two such beads of radius $R$, treated as effective particles, have a far smaller relative range of attraction than the primary particles $\left(\delta_{\text {eff }} \sim \delta / R\right)$, which for roughened surfaces may be only partly outweighed by its increased depth $\varepsilon_{\text {eff }}{ }^{5}$ For deeper quenches, the residual interaction between beads may become strong enough to cause a second round of phase separation and/or gelation at the bead level; the whole argument can be iterated to describe that case.

Ignoring this last effect for the moment, the gel region in the phase diagram is bounded by a section of liquid-gas tieline emanating from the intersection point (at $\left.\varphi_{2}\right)$ of the binodal with the MCT arrest line, and a line parallel to the quench route, emanating from this tieline at the volume fraction $\phi=\phi_{c} \equiv \varphi_{1}+\Phi_{c}\left(\varphi_{2}-\varphi_{1}\right)$ corresponding to percolation. The transition across the first boundary is 'temperature driven' (or more accurately $\varepsilon$ driven) and governed by the MCT arrest of the dense phase; quenching by this route, some features of the MCT transition (which applies directly only for $\phi \geqslant \varphi_{2}$ ) are expected to be detectable dynamically at much lower concentrations [16]. In contrast, the 'pressure-driven' (or more accurately, $\phi$-driven) transition across the remaining section of the gel boundary should fall into the percolation universality class [20]. The possible secondary gelation of arrested beads at deep quenches complicates this picture somewhat; we have neglected it in the figure and do not pursue this here.

Rapid quench scenario. Rapid quenches are ones in which the system finds itself under conditions of strong, quasi-irreversible aggregation, so that bonds created in collisions between

\footnotetext{
5 Note that for ideal spheres $\varepsilon_{\text {eff }}$ would typically increase linearly with size and thus over-compensate the effect of the reduction in the effective range $\delta_{\text {eff }}$. However, the bead surfaces are expected to be roughened due to particle deposition in the ongoing quench, so that estimating the effective attraction is a subtle task left for future work.
} 
particles are very long lived. In this case one expects at low volume fractions a nontrivial episode of structure formation, akin to irreversible diffusion-limited aggregation, on a timescale fast compared to other processes. Morphologically, rapid quenches should differ from the slow quenches described above, with a more ramified local structure and a correspondingly larger region in which an arrested phase percolates; the gel phase thus extends to lower volume fractions than in a slow quench.

The resulting type-III gels are conceptually distinct from the rest, and may have some intriguing properties. Their analysis is more difficult, as can be appreciated by considering a deep rapid quench at low volume fractions. This is the case considered in the CMCT theory of [16]. The basic idea of CMCT is to allow for strong, quasi-irreversible bonding at short length scales by applying MCT, not to primary particles, but to fractal aggregates created by such bonding. This is quite similar to the idea already introduced above, of iterating MCT to describe the possible gelation and arrest of dense beads. However, the calculation of the effective particle parameters is even more subtle and, in particular, allows for an entropic decrease in the effective attraction between clusters. This reflects the multiplicity of internal bonds at which two clusters, having joined to form a larger one, can now be broken apart to recover two clusters of similar size and shape to the original pair [16]. Another potentially important feature is the ability of CMCT to allow for a build-up of long-range repulsions due, for example, to a very slight Coulomb repulsion that may be present in many colloids, even in organic solvents [21]. This build-up also lowers the effective attraction strength as the clusters get larger.

The effect of the scale-dependent effective bond strength, combined with a scale-dependent range, means that in CMCT there is a tendency, as aggregation proceeds, to move away from the attraction-driven arrest scenario and towards a more conventional repulsion-driven glass. Setting aside phase separation effects (for now), the key issue in gelation is then whether the effective attractions become small while the volume fraction of clusters is still fairly low, or whether, by the time they stop aggregating, the clusters are dense enough to be arrested anyway by repulsive caging. In the first case one predicts a semi-ergodic phase comprising a fluid of clusters [22], in the second an arrested cluster phase which is nonergodic at all scales and thus a gel [16].

For type-III gels the interplay with phase separation is quite complicated; roughly speaking it follows the lines already developed above for slow quenches, but with CMCT-like arrest replacing the standard MCT arrest throughout the discussion. However the phase separation itself is also perturbed by what is happening at the cluster scale, which is a further complication. (In mitigation, for many systems the dense phase will be dense enough for the CMCT and MCT predictions to nearly coincide through much of the phase diagram anyway.) Within the CMCT picture there can be two distinct forms of cluster phase: one in which there is no tendency to macroscopic phase separation but clusters stop growing due to the build-up of repulsion (Coulombic or entropic); another in which phases have separated but the dense arrested phase does not percolate [16]. The latter includes the gel beads described previously as a limiting case [14].

CMCT is based on a simplifying assumption that the timescale for internal reconstruction of clusters is slow compared to the timescale for realizing a state of repulsion-driven arrest at larger scales. This may be safe for Coulombic stabilization, but needs careful further test, against both experiment and simulation, in the case where the bond strength is effectively reduced by the entropy associated with bond breaking internal to a cluster [23].

\subsection{Combining MCT with phase separation: further remarks}

By translating knowledge of the interaction potential into a combined topographic map of the MCT transition surface and the gas-liquid coexistence, we were able to present above and 


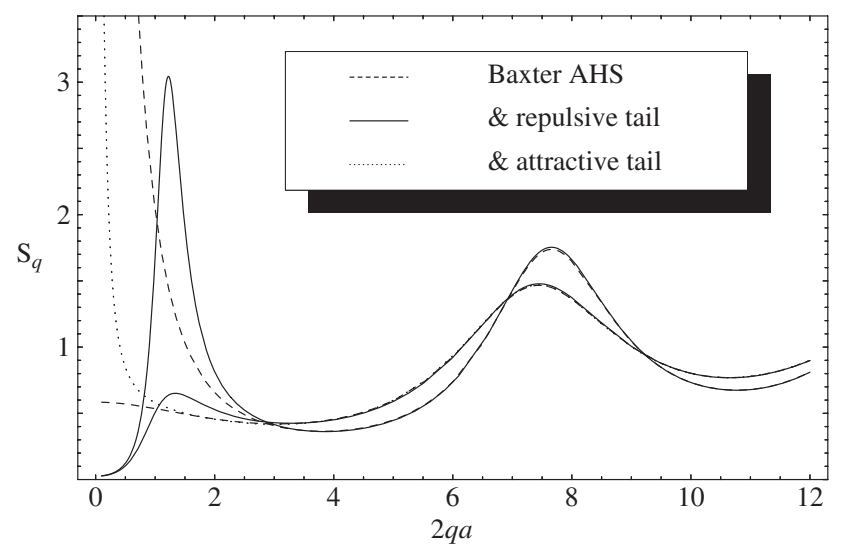

Figure 2. Static structure factor $S_{q}$ for the AHSS [24] with volume fraction $\phi=0.3$ and adhesiveness parameter $\tau=0.08$ and 0.2 , respectively (the former being closer to the spinodal and showing the more pronounced oscillations), calculated by a random phase approximation. Three cases of competing interactions are distinguished: the bare AHSS (dashed curves), the AHSS with an additional repulsive Yukawa barrier of height 0.1 and decay length 2.5 (solid curves), and with an attractive tail of the same range but with an amplitude -0.004 (dotted curve, only for $\tau=0.2$ since $\tau=0.08$ lies within the spinodal region).

in [16] (see also [14]) a guide for disentangling some of the experimentally observed complex phenomenology of attracting colloids.

We now take a somewhat broader perspective in which, on top of the hard-sphere repulsions, the pair potential can be represented as the sum of a strong short-range attraction and an additional weak long-range interaction that may either be attractive or repulsive. Numerous examples-from microemulsions, through block copolymers, to supercooled water-show that such competing interactions can give rise to complex phase behaviour. Even the adhesive hard-sphere system (AHSS) of Baxter [24] is now known to have two competing crystalline phases, two glasses, and a nontrivial re-entry between them. This stems from a competition between the hard-core repulsion and the short-range attraction [6, 25]. Adding further longrange interactions can certainly complicate this further.

Insofar as it destabilizes the equilibrium liquid, the origin of this complexity is manifest in the static structure factor $S_{q}$ [26]; this is also the input for MCT calculations. In figure 2, $S_{q}$ is shown for an AHSS with additional long-range interaction. The low- $q$ tail of the structure factor shows the characteristic upturn found near any spinodal. A sharper upturn is seen (well before the spinodal is reached) if a very weak but longer-ranged attraction is added (dotted). In contrast, adding a repulsive barrier can energetically prohibit macroscopic phase separation as evidenced by the suppression of the spinodal divergence and the shifting of the new relative maximum in $S_{q}$ to finite wavevectors $q=q_{c}$ (solid curves). Near the former spinodal, the new peak grows in height without bound, thus heralding microphase separation, in which the homogeneous fluid decomposes into finite, self-limiting domains of liquid-like and gaslike character. The domain size $\left(\simeq q_{c}^{-1}\right)$ is controlled by (and roughly scales as) the range of the extra repulsion.

To predict the nonequilibrium behaviour in the presence of the competing interactions, note first that thermodynamics and kinetic arrest are sensitive to different features of the pair potential. Gas-liquid phase separation can be induced by a weak long-ranged attraction, as familiar from van der Waals' theory; and similarly, even finite repulsions may cause (Wigner) crystallization if sufficiently long ranged. Attraction-driven arrest, on the other hand, 
relies on kinetic trapping of a thermodynamically stable fluid by short-ranged forces $(\delta \ll 1)$, and according to MCT the arrest is triggered by the high Fourier modes $q a \simeq \delta^{-1} \gg 1$, as encoded in the direct correlation function $c_{q} \propto 1-S_{q}^{-1}$ [1]. This contrasts with the crowding of the nearest-neighbour shell visible in the first peak of $S_{q}$ near $q a \simeq \pi$, which leads to the 'cage effect' for repulsive hard-sphere glasses.

Recently there has been much debate whether the long-range repulsion itself [27], or the frustration resulting from the competing interactions [28], may be a new driving mechanism for the formation of more exotic arrested states. Indeed, apart from the repulsive (and attractive) hard-core glasses familiar in colloids, MCT allows for another transition scenario triggered by long-range repulsions that give rise to a dilute arrested state, which was interpreted as a Wigner glass [29]. Even liquid-crystalline domains of asymmetric particles have been predicted to undergo kinetic arrest [30]. Along somewhat different routes similar conclusions were reached about so-called stripe glasses in microemulsions and related systems [28]. Several of the mentioned mechanisms (attractive/repulsive 'hard-core' interactions of arrested spinodal textures; a glass transition triggered by a microphase peak in $S_{q}$; the Wigner glass) may be relevant to the possible gel phases of Coulomb-stabilized cluster fluids [21, 22]. More generally we therefore expect that the interplay of MCT with microphase separation could lead to a phenomenology at least as rich and interesting as the one elaborated above for the case of bulk phase separation.

\section{Simulation of dense attractive colloids}

We now turn to our second theme, which is to test the predictions of MCT for dense attractive colloids, in a regime of concentration and interactions where these predictions could be reasonably expected to work. Thus we can bypass the various complications connected with phase separation and aggregation that arise at low density, as were considered in section 2.

\subsection{Simulation details}

Newtonian dynamics simulations were performed to test the theoretical predictions on the gel transition in a system whose pair potential mimics the depletion attraction found in colloidpolymer mixtures [6]. One thousand polydisperse particles were considered in the canonical ensemble. The core-core repulsion between particles is given by $V_{\mathrm{sc}}(r)=k_{\mathrm{B}} T\left(r / a_{12}\right)^{-36}$, where $a_{12}=a_{1}+a_{2}$, with $a_{1}$ and $a_{2}$ the radii of the particles. A flat distribution of radii was used to prevent crystallization, with a (half-)width equal to one-tenth of the mean radius, $\Delta=0.1 a$. The interaction between the colloidal spheres is given by the Asakura-Oosawa interaction potential, which considers the depletion of ideal polymers [31], corrected to take into account the polydispersity of the colloids [32]. The total potential ( $\left.V_{\mathrm{tot}}=V_{\mathrm{AO}}+V_{\mathrm{sc}}\right)$ was corrected close to $a_{12}$ to ensure that the minimum of the total potential is at $a_{12}$ [33].

A long-range repulsive barrier was added to the interaction potential in order to prevent liquid-gas separation at high attraction strength. When this barrier is allowed for, the potential is attractive at short distances, and slightly repulsive at longer distances. The range of the attraction is given by the size of the polymers, $\xi$, and the strength at contact, $r=a_{12}$, is proportional to the polymer volume fraction $\phi_{\mathrm{p}}$. The height of the repulsive barrier is $1 k_{\mathrm{B}} T$, whereas arrest occurs for attraction strengths above $\sim 7 k_{\mathrm{B}} T$ [33]. While the remarks of section 2.3 serve as a warning that this kind of barrier could influence the results, our aim here is merely to avoid the liquid-gas separation that would otherwise complicate matters considerably. The barrier is thus chosen as small as is consistent with achieving this.

In our simulations, lengths are measured in units of the mean particle radius, $a$, and time in units of $\sqrt{4 a^{2} / 3 v^{2}}$, with $v$ the thermal velocity, which is set to $\sqrt{4 / 3}$. The equations of motion 


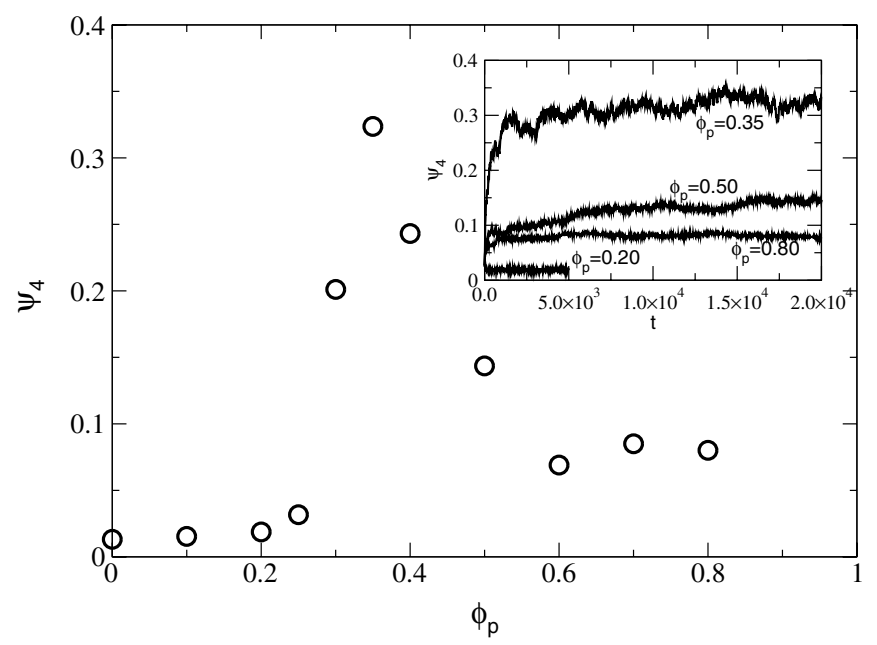

Figure 3. Long-time limit of the demixing order parameter, $\psi_{4}$, as a function of the polymer volume fraction, $\phi_{\mathrm{p}}$. Inset: evolution of $\psi_{4}$ with time for different $\phi_{\mathrm{p}}$ as labelled.

were integrated using the velocity-Verlet algorithm, with a time step of 0.0025 . The volume fraction of colloids is fixed at $\phi=0.40 ; \xi=0.1 a$; and the attraction strength is parametrized by the polymer volume fraction, $\phi_{\mathrm{p}}$.

\subsection{Suppression of phase separation}

Separation of the system into two phases of different density was monitored by a demixing order parameter, $\psi_{4}$. The system is divided in $4^{3}$ boxes of equal size, and the deviation of the density in every box with respect to the average is measured by this parameter; a homogeneous system shows $\psi_{4}$ close to zero and a phase-separated system has a much bigger value. With this parameter, the isochore $\phi=0.40$ was first studied for the system without a long-range barrier,in order to see any effect gelation might have on liquid-gas separation (and, potentially, vice versa).

The inset to figure 3 shows the temporal evolution of $\psi_{4}$ for different polymer fractions. At low $\phi_{\mathrm{p}}$, the system is homogeneous, i.e., the attraction strength is too low to induce liquidgas separation, and for $\phi_{\mathrm{p}}=0.35$, the system demixes, as shown by the increase of $\psi_{4}$. At even higher attraction strengths, the system does not phase separate into denser and more dilute phases, as expected, but becomes again more homogeneous. The long-time limit of the separation parameter, which is plotted in figure 3 as a function of $\phi_{\mathrm{p}}$, captures this scenario. The liquid-gas transition is found to take place at $\phi_{\mathrm{p}}=0.30$, in agreement with previous simulations [34]. The $\psi_{4}$ parameter does not increase monotonically, as expected from equilibrium thermodynamics, but reaches a maximum and decreases. A plateau at $\psi_{4} \approx 0.08$ is observed above $\phi_{\mathrm{p}}=0.60$, indicating a quasi-homogeneous system. We may thus conclude that some mechanism must be present that hinders liquid gas separation at high attraction strength and finally prevents it. This mechanism is gelation, i.e., attraction-driven arrest.

\subsection{Results for averaged quantities}

To analyse the arrest mechanism, the long-range repulsive barrier was restored to the interaction potential, thus forbidding liquid-gas separation and allowing the gelation to be probed in its own right. With the barrier, the system is macroscopically homogeneous at all polymer fractions 


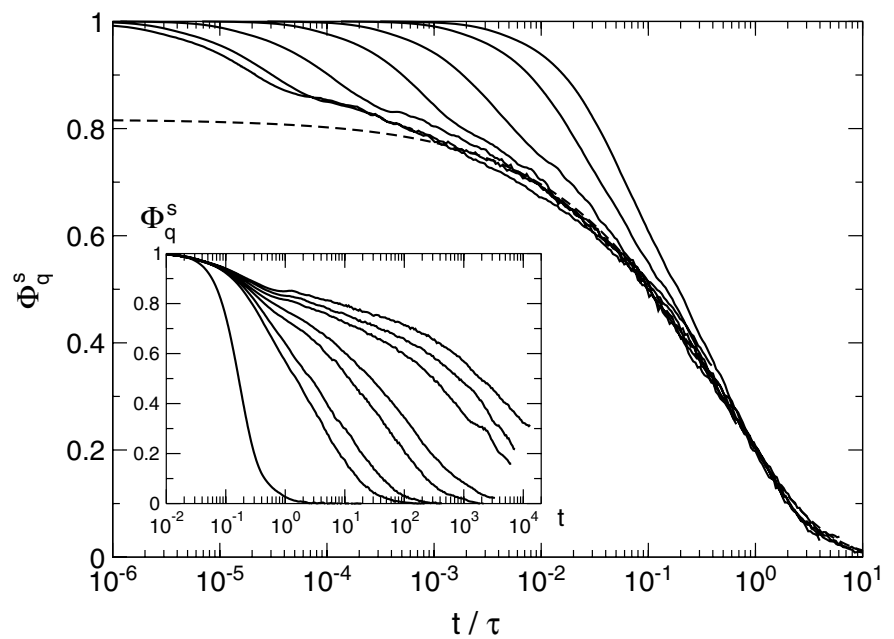

Figure 4. Density-density correlation functions for increasing polymer fraction, rescaled at long times. From left to right $\phi_{\mathrm{p}}=0.425,0.42,0.415,0.41,0.40,0.39$ and 0.375 , and Kohlrausch fitting to the $\alpha$-decay (dashed curve). Inset: unscaled correlation functions for the same states, from right to left. The hard-sphere case, $\phi_{\mathrm{p}}=0$ is added (left-hand curve).

studied, although it presents detectable voids and 'tunnels' when viewed from certain angles, and also shows a low-angle peak in the structure factor at $q a \sim 1$ [33]. However, this is always lower than the primary near-neighbour peak at $q a \sim \pi$.

The dynamics of the system close to gelation is studied by means of the self part of the density-density correlation function, i.e. $\Phi_{q}^{s}(t)=\left\langle\exp \left\{\mathbf{i q} \cdot\left[\mathbf{r}_{j}(t)-\mathbf{r}_{j}(0)\right]\right\}\right\rangle$, where the brackets indicate averaging over particle $j$ and time origin, and $\mathbf{q}$ is the wavevector. Figure 4 shows the density correlator for increasing polymer fraction for $q a=6.9$, the second (nonmicrophase) peak in the structure factor. The two-step decay in the correlation functions is similar to the behaviour of states approaching the glass transition in the Lennard-Jones system (LJS) or in hard spheres (HS) $[35,36]$. Furthermore, the decay from the plateau can be rescaled at long times for all the states presented (with deviations for the state closest to the transition, as seen also in other simulations $[35,36])$ and the master decay can be fitted using the Kohlrausch form, $\Phi_{q}^{s}(t)=A_{q} \exp \left(-\left(t / \tau_{K}\right)^{\beta}\right)$, a signature of MCT-like nonergodicity transitions. Similar scalings are obtained at all the wavevectors studied (not presented here).

Using the predictions from MCT, the early decay from the plateau is correctly described by the von Schweidler law: $\Phi_{q}^{s}(t)=f_{q}^{s}-h_{q}^{(1)}(t / \tau)^{b}+h_{q}^{(2)}(t / \tau)^{2 b}+\mathrm{O}\left(t^{3 b}\right)$, where $f_{q}^{s}$ is the nonergodicity parameter and $h_{q}^{(1)}$ and $h_{q}^{(2)}$ are amplitudes. All three of these are state independent (specifically, independent of $\phi_{\mathrm{p}}$ ), whereas $\tau$ is a timescale which carries statedependent information, increasing as the glass transition is approached. The von Schweidler expression correctly describes our correlation functions for all states and wavevectors, and the results from the fittings show that $f_{q}^{s}$ in this attractive case is much bigger than the nonergodicity parameters found in HS or LJS. This fact indicates that the localization length is much shorter than in those cases, showing that the driving mechanism for this transition is the formation of long-lived bonds between particles. Moreover, the von Schweidler exponent, $b$, from the fittings also differs from the HS or LJS values, yielding $b=0.37$ [33], in agreement with the predictions from MCT [2].

For the timescales $\tau_{q}$ of the $\alpha$ decay, defined by $\Phi_{q}^{s}\left(\tau_{q}\right)=f_{q}^{s} / e$, MCT predicts a powerlaw divergence, with an exponent $\gamma$ related to $b$. Figure 5 presents the timescales at different 


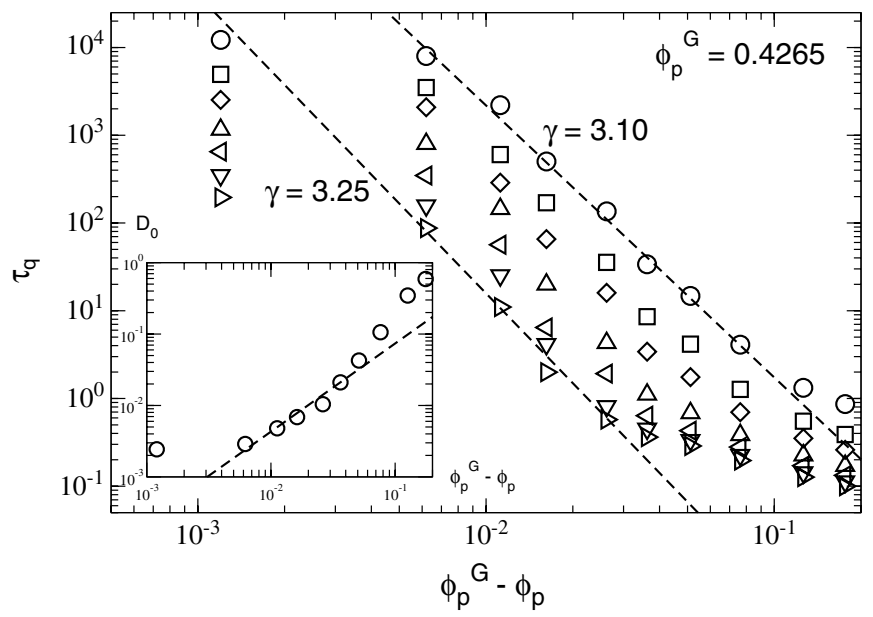

Figure 5. Wavevector-dependent timescale, $\tau_{q}$, versus $\phi_{\mathrm{p}}^{\mathrm{G}}-\phi_{\mathrm{p}}$ for different wavevectors. From top to bottom $q a=3.9,6.9,9.9,15,20,25,30$. The lines are power-law fittings. Inset: self-diffusion coefficient, $D_{\mathrm{s}}$, versus $\phi_{\mathrm{p}}^{\mathrm{G}}-\phi_{\mathrm{p}}$ and power-law fitting.

wavevectors on logarithmic axes. The power-law divergence is very clear, and the exponents agree with the value expected from $b$, which is $\gamma=3.1$. The gel point, $\phi_{\mathrm{p}}^{\mathrm{G}}$, also fitted in the analysis, is found at $\phi_{\mathrm{p}}^{\mathrm{G}}=0.4265$ for this volume fraction, $\phi=0.40$. It is interesting to note that the competition between gelation and liquid-gas separation at this polymer fraction was already apparent in our study of the system without the long-range barrier, although gelation fully impedes phase separation only well above this value of $\phi_{\mathrm{p}}$.

MCT also predicts a power-law decay, with the same exponent $\gamma$, for the self-diffusion coefficient, $D_{0}$. This coefficient is determined from the long-time behaviour of the mean squared displacement, $\left\langle\delta r^{2}\right\rangle=6 D_{0} t$. Fixing the gel point to the value reported above, the fitted exponent in this case is $\gamma=1.23$, quite different from the value obtained above for the divergence of the timescale. Such differences are obtained in the analysis of other model systems, LJS or HS, and have been attributed to the presence of dynamical heterogeneities in the system. However, in our work the difference between the exponents determined from the timescale and the diffusion coefficient is larger than in these other cases. This suggests a possible stronger role for dynamic heterogeneities in the presence of short-range attractions.

\subsection{Dynamical heterogeneity}

The dynamical heterogeneities of a state can be studied by analysing the distribution of the squared displacement of particles measured between some arbitrary time $t=0$ and a later time $t=t^{*}$. A homogeneous fluid should present a single-peaked distribution, its width depending on $t^{*}$ and $D_{0}$. Figure 6 presents this distribution for different states approaching the gel transition, where $t^{*}$ has been chosen so that $\left\langle\delta r^{2}\left(t^{*}\right)\right\rangle=10 a^{2}$ for all states. At low polymer fractions, the system is indeed homogeneous and the distribution of squared displacements agrees with the theoretical expectation for a system of Brownian particles. However, at higher attraction strengths, the system becomes more and more heterogeneous and the distribution more bimodal.

For polymer volume fractions above $\phi_{\mathrm{p}}=0.41$ two peaks are clearly observed in the distribution, showing one population of particles that move much less than a particle radius 


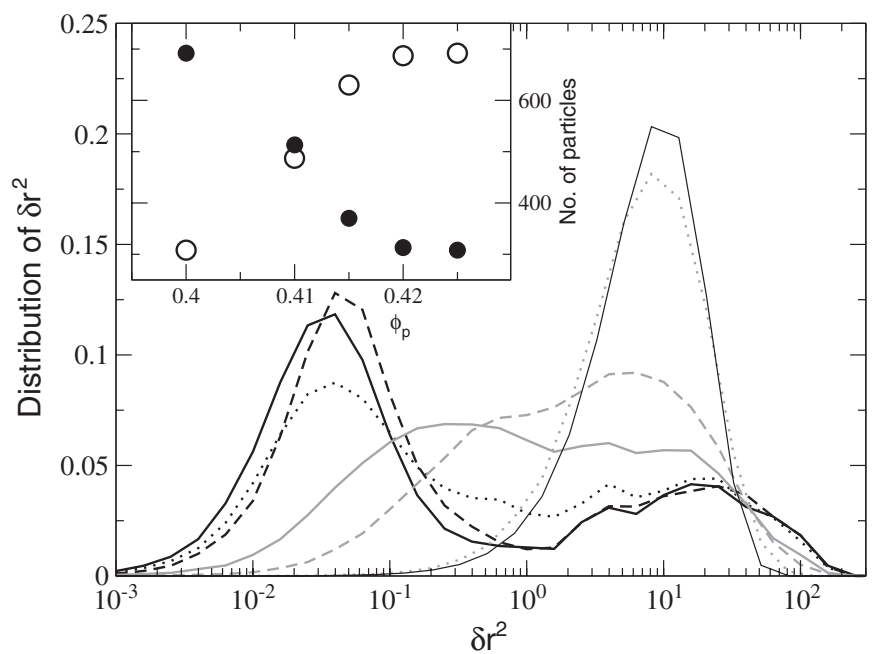

Figure 6. Distribution of squared displacement for $\phi_{\mathrm{p}}=0.30$ (grey dotted curve), $\phi_{\mathrm{p}}=0.40$ (grey dashed curve), $\phi_{\mathrm{p}}=0.41$ (grey solid curve), $\phi_{\mathrm{p}}=0.415$ (black dotted curve), $\phi_{\mathrm{p}}=0.42$ (black dashed curve), and $\phi_{\mathrm{p}}=0.425$ (black solid curve). In all cases $\left\langle\delta r^{2}\right\rangle=10 a^{2}$. The thin black curve is the theoretical distribution for a system of noninteracting Brownian particles. Inset: number of slow particles (open circles) and fast particles (closed circles) in the system as a function of the polymer volume fraction.

and another population of very mobile particles. (These of course combine such that the average squared displacement is $\left\langle\delta r^{2}\right\rangle=10 a^{2}$, since this was used to define $t^{*}$.) In fact, these two populations of particles can be distinguished at all times. The exchange between them is detectable, but very slow, allowing an analysis of the system to proceed as if it were composed of two entirely distinct populations: 'fast' particles and 'slow' ones [37].

The nontrivial structure of the incipient gel (with voids and tunnels) makes possible the existence of a subset of particles fully integrated into a percolating gel-like structure, each with a large number of neighbours, while also allowing another subset of particles to be present at the surface of the same structure and thus to have only a few neighbours. The slow particles form a stiff structure which is very slow to relax, whereas the fast particles surround this structure, with a high mobility. Since there is a relatively large amount of free space in the system, the fast particles move freely and not in clusters or in stringlike motions. The fast particles attach from time to time to the incipient gel structure at surface sites but generally depart again before getting trapped. Preferential sites for this transient adsorption of fast particles are detected in pockets of the incipient gel formed by the slow ones, where they can establish more bonds with slow particles [37].

\subsection{Implications for MCT}

It is interesting to point out that the average behaviour of the system is correctly described by MCT [33], both in the universal properties and the specific predictions for the attraction driven glass transition. The main exceptions to this involve the non-Gaussian parameter (not shown; see [37]) and the discrepancy between $\gamma$ as measured from diffusion and from structural relaxation. However, the detailed dynamics, involving two concurrent populations of particles, signifies very strong dynamical heterogeneities which are not really consistent with the approximation scheme lying behind MCT calculations. The strong DH observed 
in our incipient colloidal gels is related, it appears, to structural heterogeneities which have no direct analogue in the glass transition driven by repulsion [10]. At lower densities, these heterogeneities are even more important; in that region one expects to require significant modifications to MCT, as developed and discussed in section 2.

\section{MCT under shear}

The framework of MCT can be extended to address the relationship between stress and strainrate in a system undergoing shear [9, 38, 39]; this includes a way to relate the yield stress of an arrested phase to its static structure factor $S_{q}$ as determined in an unstrained state. Via $S_{q}$, the effects of attractive interactions on the nonlinear stress response can be incorporated. The response is always shear thinning. A schematic extension of this approach, allowing for a stress-dependent MCT vertex, admits the possibility of shear thickening states, which are often observed experimentally in dense repulsive colloids [40]. Although we expect attractive interactions also to have interesting effects on shear-thickening, we focus below on the shearthinning case.

The approach of $[9,38]$ predicts a finite yield stress $\sigma^{+}$at the arrest transition. For gels of attracting colloids, the scaling of $\sigma^{+}$with the width of the attraction is found below by combining a virial-type analysis of the AHSS model [41] with the model of isotropically sheared hard spheres' (ISHSM) [38, 39]. (This model isotropizes advection in its effect on density fluctuations.) The resulting scaling can be compared to the value $\sigma^{+}=0.75 k_{\mathrm{B}} T / a^{3}$ found at the glass transition of the ISHSM [39].

The ISHSM derives the nonlinear flow curves (namely, stress $\sigma$ versus strain rate $\dot{\gamma}$ ) from the competition between the slowing down of the structural dynamics captured in the classical MCT, and the speeding up of fluctuations caused by shear advection. The latter mechanism has been intensively studied in the context of fluctuations in sheared systems close to criticality [42], and close to ordering or microphase transitions [43]. It causes a time dependence of the wavevector of an arbitrary fluctuation

$$
\mathbf{q}(t)=\left(q_{x}, q_{\mathrm{y}}+q_{x} \dot{\gamma} t, q_{z}\right),
$$

where the shear is along the $x$-direction with $v_{x}=\dot{\gamma} y ; t$ here is the time since the start of shearing (or the birth of a fluctuation). If a fluctuation initially has a wavelength $\sim 1 / q$, at a later time $t$ its wavelength $\sim|\mathbf{q}(t)|^{-1}=1 / q(t)$ will be smaller, leading to decay of the fluctuation caused by fast, small-scale particle rearrangements.

The scaling of the yield stress $\sigma^{+}$with the attraction range $\delta$ can, for small $\delta$, be estimated from a virial expansion of $S_{q}$ for the AHSS: one finds $1-S_{q}^{-1} \rightarrow 6 A \phi \delta \sin (2 q a) /(q a)$, where $A=\exp \varepsilon-1$. Inserting this expression into the ISHSM [39], one can take the limit $\phi \rightarrow 0$ and $A \rightarrow \infty$ with $\Gamma_{v}=6 \phi A^{2} \delta / \pi^{2}$ held fixed. This leads to the longitudinal memory kernel:

$$
m_{\tilde{q}}(t) \rightarrow \frac{\Gamma_{v}}{2 \tilde{q}^{2}} \int^{\tilde{k}_{>}} \mathrm{d}^{3} \tilde{k}\left(\frac{\tilde{\mathbf{q}} \cdot \tilde{\mathbf{k}}}{\tilde{q} \tilde{k}}\right)^{2} \cos \left(\frac{\tilde{k}(t)-\tilde{k}}{\delta / 2}\right) \Phi_{\tilde{k}}(t) \Phi_{|\tilde{\mathbf{q}}-\tilde{\mathbf{k}}|},
$$

while the corresponding expression for the steady state shear stress becomes

$$
\sigma \rightarrow \dot{\gamma} \frac{k_{\mathrm{B}} T}{a^{3}} \frac{\phi \Gamma_{v}}{5 \delta^{2}} \int_{0}^{\infty} \mathrm{d} t \int_{0}^{\tilde{k}_{>}} \mathrm{d} \tilde{k} \tilde{k}^{2} \cos \left(\frac{\tilde{k}(t)-\tilde{k}}{\delta / 2}\right) \Phi_{\tilde{k}}^{2}(t) .
$$

Here, the limit $\delta \rightarrow 0$ applies, and the rescaled wavectors $\tilde{q}=q a \delta$ and a cut-off $\tilde{k}_{>}$were introduced; choosing $\tilde{k}_{>} \approx 3.68$ maps the AHSS virial results onto the corresponding results of an attractive square-well system with range $\delta$ [41]. 
In the approach of $[38,39]$, these equations are closed via an MCT-like relationship for the normalized density correlation functions $\Phi_{\tilde{q}}(t)$, whence we obtain predictions for the nonlinear rheology close to the arrest transition (at $\Gamma_{v}^{c}=1.42$ ) of attracting colloids with small $\delta$ and low $\phi$. Although the low- $\phi$ approximation appears drastic, if shear is switched off in equation (2), the results for the arrest line at small $\delta$ agree qualitatively with those of [2] up to fairly large $\phi[12,41]$. (Thus the approximations made are faithful to the standard MCT analysis, though they neglect the physics of heterogeneity discussed in section 2.) With shear present, we can now analyse the altered behaviour of $\Phi_{\tilde{q}}(t)$ at the onset of arrest and then compute the limiting stress at low shear rate by inserting the resulting asymptotics into equation (3) [39]. This gives a finite yield stress $\sigma^{+}$just within the glass, which falls abruptly to zero on entering the fluid.

Asymptotically the $\Phi_{\tilde{q}}(t)$ obey a 'yielding scaling law', $\Phi_{\tilde{q}}(t) \rightarrow \Phi_{\tilde{q}}^{+}(\hat{t})$ with $\hat{t}=t / \tau(\dot{\gamma})$ and $\tau(\dot{\gamma})$ a shear-rate dependent characteristic time. Also one finds the limiting closure relation [39]:

$$
\Phi_{\tilde{q}}^{+}(\hat{t})=m_{\tilde{q}}^{+}(\hat{t})-\frac{\mathrm{d}}{\mathrm{d} \hat{t}} \int_{0}^{\hat{t}} \mathrm{~d} t^{\prime} m_{\tilde{q}}^{+}\left(\hat{t}-t^{\prime}\right) \Phi_{\tilde{q}}^{+}(\hat{t}),
$$

with $m_{\tilde{q}}^{+}(\hat{t})$ obtained from equation (2) using the limiting form $\Phi_{\tilde{q}}^{+}(\hat{t})$. The yield stress $\sigma^{+}$then follows from inserting $\Phi_{\tilde{q}}^{+}(\hat{t})$ into equation (3).

The expressions for the memory kernel and stress, equations (2) and (3), contain a rapidly oscillating term $\cos (2(\tilde{k}(t)-\tilde{k}) / \delta)$, which arises from interference of the particle density fluctuations within the narrow region of attraction. Studying this factor illuminates the role of shear. Without shear, constructive interference holds, and the factor is unity; the memory kernels describe as usual bond-formation owing to attraction, and the correlator stays arrested at its glass value, $\Phi_{\tilde{q}}^{+}(\hat{t})=\Phi_{\tilde{q}}^{+}(0)=f_{\tilde{q}}^{c}$. Under shear, the advection of wavevectors (giving $\left.\tilde{k}(t)-\tilde{k}=\left(k_{x} k_{\mathrm{y}} / k\right) \dot{\gamma} t+\cdots\right)$ produces rapid oscillations in this term when $\delta$ is small. The interference is destroyed, causing a fast decay of the memory functions. The timescale $\tau(\dot{\gamma})$ needs to be found self-consistently from equations (2) and (4); the preceding argument shows that it scales with shear-rate and attraction range as $\tau(\dot{\gamma})=c_{a} \delta /|\dot{\gamma}|$ where $c_{a}$ is of order unity. In contrast, for repulsive interactions in the ISHSM, we found [38, 39] $\tau(\dot{\gamma})=c_{r} /|\dot{\gamma}|$ with $c_{r}$ again of order unity. Integrating equation (3), one obtains (with further constants $c_{a, r}^{\prime}$ ) the scaling expressions $\sigma_{\text {AHSS }}^{+}=c_{a}^{\prime} G_{a} \delta$ and $\sigma_{\text {ISHSM }}^{+}=c_{r}^{\prime} G_{r}$ for the yield stresses at the two transitions. Here $G_{a, r}$ are the corresponding shear moduli which, within MCT, are predicted to acquire finite values on arrest. Their scalings have been discussed elsewhere [1, 2, 12, 41, 44]; for AHSS one has $G_{a} \sim k T a^{-3} \delta^{-2}$ whereas $G_{r} \simeq k T a^{-3}$ holds for hard spheres.

The final scaling result for AHSS is thus $\sigma^{+} \simeq G_{a} \delta \simeq k T a^{-3} \delta^{-1}$. This dependence of the yield stress on the attraction range leads to interesting scenarios in systems where both attraction- and repulsion-driven arrest can be observed [7, 45], and where at high enough concentrations the local structural dynamics should dominate the rheological behaviour. Interpreting the ratio of yield stress to the elastic modulus as a yield strain $u_{\mathrm{y}}=\sigma^{+} / G$, the scaling dependence of $\sigma^{+}$on $\delta$ can easily be understood. The yield strain of a (high-density) colloidal gel is of order the relative range of the attraction $\delta$ : the solid is shear melted as soon as particle bonds are broken. At the same time, the scaling of the modulus is quite different for attractive and repulsive glasses; the attractive glass/gel is much stiffer as noted above.

Thus two states of equal viscosity, close to the attractive and the repulsive branches of the arrest line, can have quite different relaxation times. It would be interesting to measure the dynamics, in the region where the two branches meet, along contours of equal zero-shear viscosity, as was done (without shear) for contours of equal diffusivity in recent simulations [46]. Because the yield strain is much smaller for a bonded glass than for a caged one, the nonlinear rheology should vary strongly along these contours. 
A simple 'generalized nonlinear Maxwell model' [39] summarizes the pertinent behaviour for both AHSS and ISHSM cases:

$$
\eta(\dot{\gamma})=\eta(\infty)+\left(\frac{1}{\eta(0)}+\frac{|\dot{\gamma}|}{\sigma^{*}}\right)^{-1} .
$$

Here $\eta(0)$ is the zero-shear viscosity; $\eta(\infty)$ is a (small) limiting viscosity at very high shear rates (presumably set by hydrodynamic interactions, ignored here); and $\sigma^{*}$ is a characteristic stress scale of the order of the yield stress discontinuity $\sigma^{+}$at the onset of arrest. The above model gives near-plastic yield in arrested systems $(\eta(0)=\infty)$ but can also describe a highly viscous but shear-thinning fluid phase [39]. In such a phase, close to a repulsive glass transition, the nonlinear viscosity $\eta(\dot{\gamma})$ decreases significantly from its zero-shear value $\eta(0)$ once $\eta(0) \dot{\gamma} a^{3} / k T$ is of order unity. In a fluid close to an attraction-driven arrest, the decrease is only at much higher shear rates, with $\eta(0) \dot{\gamma} a^{3} / k T \approx 1 / \delta$. This is associated with the much larger value of the yield stress $\left(\sigma^{+} \sim 1 / \delta\right)$, despite the much smaller value of the yield strain $\left(u_{\mathrm{y}} \sim \delta\right)$, in the nearby arrested phase.

\section{Concluding remarks}

We have described various developments in the application of MCT ideas to colloids with short-range attractions. This theory is very successful at high densities in predicting reentrant transitions induced by such attractions [7], yet this re-entrance is caused by density fluctuations whose presence partly undermines the assumptions of homogeneity on which MCT is based. For a colloid volume fraction of $\phi=0.4$ we found by simulations that the MCT predictions for averaged properties remain generally good despite a surprising degree of dynamic heterogeneity. The latter is washed out in the averages, but can be probed more closely through the distribution of mean-squared displacements, and this analysis reveals a strong partitioning of particles into fast and slow populations. At much lower volume fractions the assumption of homogeneity becomes clearly wrong, but this can be partially addressed, at least at the level of qualitative prediction, by carefully combining MCT ideas with those of phase separation (arrest of one phase in a phase-separated morphology) and/or irreversible aggregation (applying MCT at the cluster scale). The effects of short-range attractions on the rheology of colloidal suspensions are potentially quite subtle. The preliminary calculations reported above already show some interesting trends, particularly for the range dependence of the yield stress in the attraction-driven glass, and for the shear-thinning behaviour in the nearby fluid phase.

\section{Acknowledgments}

Financial support is acknolwedged from the Ministerio de Ciencia y Tecnología under project MAT2003-03051-CO3-01 (AMP); Deutsche Forschungsgemeinschaft under grant No Fu3093 (MF); and EPSRC GR/S10377/01.

\section{References}

[1] Bergenholtz J and Fuchs M 1999 Phys. Rev. E 595706

[2] Dawson K, Foffi G, Fuchs M, Götze W, Sciortino F, Sperl M, Tartaglia P, Voigtmann Th and Zaccarelli E 2001 Phys. Rev. E 63011401

[3] Fabbian L, Götze W, Sciortino F, Tartaglia P and Thiery F 1999 Phys. Rev. E 59 R1347

[4] Götze W and Sperl M 2003 J. Phys.: Condens. Matter 15 S869

[5] Sperl M 2004 Phys. Rev. E 69011401 
[6] Sedgwick H et al 2004 J. Phys.: Condens. Matter 16 S4913

[7] Pham K N, Puertas A M, Bergenholtz J, Egelhaaf S U, Moussaid A, Pusey P N, Schofield A B, Cates M E, Fuchs M and Poon W C K 2002 Science 296104

[8] Cates M E 2003 Ann. Henri Poincaré 4 (Suppl. 2) S647-61

[9] Cates M E, Holmes C B, Fuchs M and Henrich O 2003 Preprint cond-mat/0310579

[10] Weeks E R, Crocker J C, Levitt A C, Schofield A and Weitz D A 2000 Science 287627

[11] Puertas A M, Fuchs M and Cates M E 2002 Phys. Rev. Lett. 88098301

[12] Bergenholtz J, Poon W C K and Fuchs M 2003 Langmuir 194493

[13] Poon W C K and Haw M D 1997 Adv. Colloid Interface Sci. 7371

[14] Sedgwick H et al 2003 Preprint cond-mat/0309616

[15] Vicsek T 1992 Fractal Growth Phenomena 2nd edn (Singapore: World Scientific)

[16] Kroy K, Cates M E and Poon W C K 2004 Phys. Rev. Lett. 92148302

[17] Del Gado E, Fierro A, de Arcangelis L and Coniglio A 2003 Europhys. Lett. 631

[18] Dickinson E 1992 An Introduction to Food Colloids (Oxford: Oxford University Press)

[19] Owen A J and Jones R A L 1998 Macromolecules 317336

[20] Lironis G, Heermann D W and Binder K 1990 J. Phys. A: Math. Gen. 23 L329

[21] Yethiraj A and van Blaaderen A 2003 Nature 421513

[22] Segrè P N, Prasad V, Schofield A B and Weitz D A 2001 Phys. Rev. Lett. 866042

[23] Zaccarelli E, Foffi G, Sciortino F and Tartaglia P 2003 Phys. Rev. Lett. 91108301 Sciortino F, Mossa S, Zaccarelli E and Tartaglia P 2003 Preprint cond-mat/0312161 Zaccarelli E, Sciortino F, Buldyrev S V and Tartaglia P 2003 Preprint cond-mat/0310765

[24] Baxter R J 1968 J. Chem. Phys. 492770

[25] Sciortino F 2002 Nat. Mater. 1145

[26] Sear R P and Gelbart W M 1999 J. Chem. Phys. 1104582

[27] Klein W, Gould H, Ramos R A, Clejan I and Mel'cuk A I 1994 Physica A 205738

[28] Westfahl H, Schamlian J and Wolynes P G 2001 Phys. Rev. B 6417403

[29] Bosse J and Wilke S D 1998 Phys. Rev. Lett. 801260

[30] Letz M, Schilling R and Latz A 2000 Phys. Rev. E 625173

[31] Asakura S and Oosawa F 1954 J. Chem. Phys. 221255

[32] Méndez-Alcaraz J M and Klein R 2000 Phys. Rev. E 614095

[33] Puertas A M, Fuchs M and Cates M E 2003 Phys. Rev. E 67031406

[34] Dijkstra M, van Roij R and Evans R 1999 Phys. Rev. E 595744

[35] Kob W and Andersen H C 1995 Phys. Rev. E 514626

[36] Kob W and Andersen H C 1995 Phys. Rev. E 524134

[37] Puertas A M, Fuchs M and Cates M E 2004 J. Chem. Phys. at press

[38] Fuchs M and Cates M E 2002 Phys. Rev. Lett. 89248304 See also Miyazaki K and Reichman D R 2002 Phys. Rev. E 66050501

[39] Fuchs M and Cates M E 2003 Faraday Discuss. 123267 Fuchs M and Cates M E 2003 J. Phys.: Condens. Matter 15 S401

[40] Holmes C B, Fuchs M and Cates M E 2003 Europhys. Lett. 63240

[41] Bergenholtz J, Fuchs M and Voigtmann T 2000 J. Phys.: Condens. Matter 126575

[42] Onuki A and Kawasaki K 1979 Ann. Phys. 121456

[43] Cates M E and Milner S T 1989 Phys. Rev. Lett. 621856

[44] Shah S A, Chen Y-L, Schweizer K S and Zukoski C F 2003 J. Chem. Phys. 1178747

[45] Eckert T and Bartsch E 2002 Phys. Rev. Lett. 89125701

[46] Foffi G, Dawson K A, Buldyrev S V, Sciortino F, Zaccarelli E and Tartaglia P 2002 Phys. Rev. E 65050802 